\section{Combining corneal hysteresis with central corneal thickness and intraocular pressure for glaucoma risk assessment}

D Pensyl', M Sullivan-Mee', M Torres-Monte', K Halverson ${ }^{1}$ and C Qualls ${ }^{2}$

\begin{abstract}
Purpose To determine whether adjusting corneal hysteresis $(\mathrm{CH})$ values for central corneal thickness (CCT) and intraocular pressure (IOP) improves its capability to differentiate primary open-angle glaucoma (POAG) from ocular hypertension (OH). Methods This prospective, observational, cross-sectional study included 169 eyes of 169 subjects with a diagnosis of POAG $(n=81)$ or OH $(n=88)$. We utilized the Ocular Response Analyzer (ORA), Pascal Dynamic Contour Tonometer (DCT), Goldmann applanation tonometer (GAT), and ORA ultrasound pachymeter to obtain $\mathrm{CH}$, IOP, and CCT values. Correlational, regression, and $t$-test analyses were conducted before and after the sample was divided into low, intermediate, and thick CCT subgroups.

Results In the full sample, $\mathrm{CH}$ and CCT were moderately correlated $(r=0.44$, $P<0.001)$. Although both were related to diagnosis in univariate regression analysis, only $\mathrm{CH}$ was independently related to glaucoma diagnosis in multivariate analysis. After the sample was divided into CCT tertiles, $\mathrm{CH}$ was significantly lower in POAG vs $\mathrm{OH}$ eyes within all three $\mathrm{CCT}$ subgroups, and $\mathrm{CH}$ was the only multivariate variable that differentiated POAG from $\mathrm{OH}$ in each CCT subgroup. Moreover, the relationship between $\mathrm{CH}$ and diagnosis was more robust within the CCT subgroups compared with the full sample, suggesting that integrating CCT into $\mathrm{CH}$ interpretation is beneficial. Adjusting $\mathrm{CH}$ for IOP did not aid diagnostic precision in this study.
\end{abstract}

Conclusion Our findings suggest that combining $\mathrm{CH}$ and CCT for glaucoma risk assessment improves diagnostic capability compared to using either factor alone. Conversely, adjusting $\mathrm{CH}$ for IOP provided no clear clinical benefit in this study. Eye (2012) 26, 1349-1356; doi:10.1038/eye.2012.164; published online 10 August 2012

Keywords: corneal hysteresis; central corneal thickness; glaucoma; ocular hypertension; Ocular Response Analyzer

\section{Introduction}

Recent research has provided evidence that corneal biomechanical properties may have a meaningful influence not only on intraocular pressure (IOP) measurements ${ }^{1-11}$ but also on the ocular effects of IOP. ${ }^{1-3,12-22}$ Central corneal thickness (CCT) has received the most attention in this regard, spurred by findings from the Ocular Hypertension Treatment Study (OHTS) in which CCT was the strongest independent predictor of conversion from ocular hypertension $(\mathrm{OH})$ to primary open-angle glaucoma (POAG). ${ }^{22}$ Furthermore, the OHTS investigators reported that after subjects were split into thin, intermediate, and thick CCT subgroups, subjects in the thinnest CCT subgroup were more than three times as likely to develop glaucoma compared with subjects in the thicker CCT subgroup. ${ }^{22}$ These results were independent of any CCT effect on IOP, and led to the inclusion of CCT measurement as a standard of care for patients with $\mathrm{OH}$. However, while CCT is a useful clinical parameter,
${ }^{1}$ Raymond G Murphy VA Medical Center, Albuquerque, NM, USA

${ }^{2}$ Department of Mathematics and Statistics, University of New Mexico School of Medicine, Albuquerque, NM, USA

Correspondence: D Pensyl, Raymond G Murphy VA Medical Center, Eye Clinic (112A), 1501 San Pedro SE, Albuquerque, NM 87108, USA

Tel: +15052651711 ext. 4229;

Fax: +1505 2565761 . E-mail: denise.pensyl@ va.gov

Received: 20 January 2012 Accepted in revised form: 14 June 2012 Published online: 10 August 2012

This work has not been previously presented. 
its sensitivity and specificity for differentiating POAG from $\mathrm{OH}$ eyes remains limited by the substantial overlap of CCT measures between these groups.

Corneal hysteresis $(\mathrm{CH})$, as measured by the Ocular Response Analyzer (ORA; Reichert Ophthalmics, Depew, NY, USA), is a more recently described corneal biomechanical parameter. $\mathrm{CH}$ is thought to reflect corneal viscoelasticity and has been described as the cornea's ability to dampen and/or buffer fluctuations in IOP. ${ }^{1}$ It is proposed that eyes with a higher $\mathrm{CH}$ tend to have more capacity to cushion short- and long-term increases of IOP, imparting a physiologically protective property. ${ }^{1,23}$ Conversely, it has been suggested that low $\mathrm{CH}$ might be expected to increase the risk for developing glaucomatous optic neuropathy, possibly due to reduced capacity of the eyewall to dampen IOP spikes ${ }^{23,24}$ and/or reduced ability of optic nerve structures to suitably respond to IOP fluctuations. ${ }^{1,25,26}$ This premise is supported by several studies, which indicate that eyes with POAG exhibit lower $\mathrm{CH}$ compared with normal eyes $^{1-3,12-16}$ and eyes with OH.1,12 In addition, lower $\mathrm{CH}$ has been linked to both glaucoma progression ${ }^{17,18}$ and severity. ${ }^{19,27}$ Furthermore, lower $\mathrm{CH}$ appears to be associated with reduced optic nerve compliance during IOP alterations. ${ }^{15}$ Taken together, these findings suggest that $\mathrm{CH}$ carries unique promise for enhancing our understanding of glaucoma pathophysiology and for improving its clinical management.

However, similar to CCT, CH exhibits considerable inter-individual variation with substantial overlap of values between diagnostic groups. ${ }^{1-3,12-16}$ Accordingly, identification of a specific $\mathrm{CH}$ value that provides good sensitivity and specificity for differentiating eyes with and without glaucoma has been elusive. Because it is a relatively dynamic variable, however, adjusting $\mathrm{CH}$ for the factors that influence its variability potentially may foster a more robust relationship between $\mathrm{CH}$ and clinical diagnosis. In effect, this adjustment would be expected to reduce measurement 'noise', which might then permit $\mathrm{CH}$ to more effectively differentiate POAG from $\mathrm{OH}$.

To determine the factors that contribute to $\mathrm{CH}$ measurement noise, we previously conducted a study to identify the relative effects of several ocular and systemic variables on $\mathrm{CH} .{ }^{27}$ Our findings indicate that the following independently influence $\mathrm{CH}$ : age, CCT, IOP, glycosylated hemoglobin (A1C), glaucoma diagnosis, and glaucoma severity. Among these factors, CCT and IOP each exerted twice as much effect on $\mathrm{CH}$ compared with the other four variables, with CCT directly related and IOP inversely related to $\mathrm{CH}$. These findings agree with many other reports and support accounting for these factors, particularly CCT and IOP, when interpreting $\mathrm{CH}^{1}{ }^{1-12,14}$
This study was therefore designed to examine whether the clinical utility of $\mathrm{CH}$ might be enhanced by adjusting $\mathrm{CH}$ for the effects of CCT and IOP. To accomplish this goal, we employed a three-step approach. We first divided our sample into thin, intermediate, and thick CCT subgroups because this mirrors the OHTS strategy and represents a proposed method for clinicians to employ CCT for risk assessment. ${ }^{28}$ Then, within each CCT subgroup, we compared $\mathrm{CH}$ values between POAG and $\mathrm{OH}$ eyes. By combining $\mathrm{CH}$ and $\mathrm{CCT}$ in this way, we were able to maintain the diagnostic power of CCT, while effectively correcting $\mathrm{CH}$ values for CCT. Subsequently, we derived $\mathrm{CH}$ values that were adjusted for IOP to determine the additive benefit that this adjustment might provide. By evaluating $\mathrm{CCT}, \mathrm{CH}$, and IOP in this manner, this study permitted a comparison of the diagnostic capability of unadjusted $\mathrm{CH}, \mathrm{CH}$ adjusted for CCT only, and $\mathrm{CH}$ adjusted for both CCT and IOP.

\section{Methods}

Beginning in July 2007, the authors screened patients from Albuquerque VA Medical Center eye clinic to determine eligibility for participation in a prospective, longitudinal, observational study. The inclusion criteria required that subjects be age 40 years or more and have open, normal angles in each eye on gonioscopy. The exclusion criteria included: corneal or scleral pathological conditions that could affect measurement of IOP; refractive error $\geq 5 \mathrm{D}$ or astigmatism $\geq 3 \mathrm{D}$; prior refractive, corneal, or incisional glaucoma surgery; secondary glaucoma diagnoses; and any visual field loss due to non-glaucomatous pathology (including retinal, optic nerve, or visual pathway disorders). Prior cataract surgery was not an exclusion factor, but data were eligible only if it was acquired at least 3 months post surgery. ${ }^{29}$

For this paper's cross-sectional analysis, we obtained data from the most recent visit. One eye was randomly selected from each subject for use in statistical analysis, unless only one eye met the criteria for a clinical diagnosis of POAG or $\mathrm{OH}$, in which case that eye was included. For this study, we required that all eyes had GAT-IOP $>21 \mathrm{~mm} \mathrm{Hg}$ on at least one prior visit. POAG eyes were characterized by glaucomatous optic neuropathy (diffuse or localized rim thinning, visible retinal nerve fiber layer (RNFL) defects, vertical cup-disc asymmetry $\geq 0.2$ that was not explained by disc size asymmetry) and repeatable glaucomatous visual field defects. The minimum criteria for glaucomatous visual field defect included glaucoma hemifield test outside normal limits on at least two consecutive reliable examinations and/or the presence of at least three contiguous test points on the pattern standard deviation 
(PSD) plot with $P<1 \%$ and at least one at $P<0.5 \%$, not including points on the edge of the field. $\mathrm{OH}$ eyes were characterized by no definitive glaucomatous optic nerve or visual field loss.

All subjects underwent comprehensive ocular examination, including slit lamp biomicroscopy, gonioscopy, ultrasound pachymetry (ORA), axial length biometry (IOL Master; Carl Zeiss Meditec, Dublin, CA, USA), dilated fundus examination with a stereoscopic assessment of the optic nerve cup-to-disc ratio (CDR), standard automated perimetry (SAP), and optical coherence tomography (OCT) of the peripapillary RNFL. SAP was performed with optimal near-point correction using the Humphrey Visual Field Analyzer II, 24-2 SITAstandard program (Carl Zeiss Meditec). For inclusion, visual fields were required to meet reliability criteria (false positives $<15 \%$ and fixation losses $<33 \%$, unless gaze-tracking demonstrated steady fixation, in which case fixation was deemed acceptable). For statistical analyses, we used the two most recent, reliable visual field tests, and calculated average values for mean defect (MD) and PSD. We acquired RNFL images with the Stratus OCT (Carl Zeiss Meditec) using the fast-RNFL circumpapillary scan pattern and only included images with good quality (scan score $\geq 6$ ). We used electronic medical record chart review to determine age, A1C, and concurrent use of IOP-lowering medication.

We employed the ORA to obtain $\mathrm{CH}$ and the instrument's other three output variables (corneal resistance factor (CRF), corneal-compensated IOP (IOPcc), and Goldmann-correlated IOP (IOPg)). Because bi-directional air jet tonometry is susceptible to corneal surface disruption, we performed this method first, before topical anesthetic use. We acquired 3-4 good quality readings for each eye and used mean values for statistical analysis. Good quality profiles were defined by relatively equal, well-defined inward and outward applanation spike heights that were located above the pressure curve, in conjunction with relatively smooth infrared reflectance signals.

After the ORA measurements, we obtained Pascal Dynamic Contour Tonometry (DCT-IOP; Ziemer Ophthalmics, Port, Switzerland) and GAT (GAT-IOP; Haag-Streit, Bern, Switzerland) in randomized order as determined by a random number generator. For DCT, we made two consecutive measurements and only accepted readings with adequate quality values (Q1-Q3). For GAT, we obtained two consecutive measurements with standard end points, unless the difference between the measurements was $>2 \mathrm{~mm} \mathrm{Hg}$, in which case we obtained an additional measurement. For all GAT measurements, an assistant pre-set the drum to between 8 and $10 \mathrm{~mm} \mathrm{Hg}$, and then recorded all measurements (in whole numbers) so that the examiner remained masked to the results. We used mean IOP values for statistical analysis. GAT calibration was confirmed before study initiation and then checked weekly per standard protocol; ORA and DCT are self-calibrating and thus no specific calibration checks were required for those instruments.

\section{Statistical analysis}

After computing the Pearson correlations between CCT, CH, GAT-IOP, and DCT-IOP for the full sample and POAG and $\mathrm{OH}$ eyes separately, Student's $t$-tests, univariate logistic regression, and stepwise multivariate logistic regression analyses were conducted to evaluate relationships between diagnosis and the study variables within the full sample. We used DCT-IOP as the IOP predictor variable within regression analyses as it is least affected by corneal characteristics. ${ }^{27,30}$

Subsequently, we divided the sample into thin, intermediate, and thick CCT subgroups, and repeated Student's $t$-tests and regression analyses within each of these subgroups. Regression equations were calculated to create IOP-adjusted $\mathrm{CH}$ variables for both GAT-IOP and DCT-IOP; these adjusted CH values were sequentially used in place of the unadjusted $\mathrm{CH}$ value previously used in CCT subgroup regression analyses. Statistical significance was defined as $P<0.05$, and all statistical analyses were performed in conjunction with a professional biostatistician (CQ) using SAS (Version 9.2; SAS Institute Inc., Cary, NC, USA). The study adhered to the tenets of the Declaration of Helsinki and all subjects completed informed consent before study participation.

\section{Results}

Of the 169 study participants, 81 met the criteria for POAG diagnosis and 88 met the criteria for $\mathrm{OH}$ diagnosis. Demographic characteristics of the sample are listed in Table 1. CCT and CH were moderately correlated in the full sample $(r=0.44, P<0.001)$, POAG group ( $r=0.37, P<0.001)$, and $\mathrm{OH}$ group $(r=0.35$, $P<0.001)$. DCT-IOP and $\mathrm{CH}$ were inversely correlated in the full sample $(r=-0.21, P=0.007)$, POAG group $(r=-0.37, P<0.001)$, and $\mathrm{OH}$ group $(r=-0.36$, $P<0.001)$. GAT-IOP and $\mathrm{CH}$ were also inversely correlated in the full sample $(r=-0.17, P=0.03)$, POAG group $(r=-0.31, P=0.005)$, and $\mathrm{OH}$ group ( $r=-0.35, P<0.001$ ) (see Table 2$)$.

In the full sample, Student's $t$-test showed that mean age, CCT, CH, CRF, A1C, IOP, concurrent IOP-lowering treatment, and variables used to determine glaucoma diagnosis (CDR, RNFL, MD, PSD) were different in POAG compared with $\mathrm{OH}$ (Table 3). Univariate and multivariate regression analysis were performed, and 
Table 1 Descriptive statistics for categorical variables $(n=169)$

\begin{tabular}{lrr}
\hline & $\mathrm{N}$ & $\%$ \\
\hline $\begin{array}{l}\text { Glaucoma-related diagnosis } \\
\text { POAG }\end{array}$ & 81 & 47.9 \\
OH & 88 & 52.1 \\
& & \\
Treatment with topical anti-glaucoma medications & & \\
POAG & 52 & 64.2 \\
$\quad$ Total & 48 & $90.6^{\mathrm{a}}$ \\
$\quad$ Prostaglandin analog & & \\
OH & 23 & 26.1 \\
$\quad$ Total & 21 & $91.3^{\mathrm{a}}$ \\
$\quad$ Prostaglandin analog & & \\
$\quad$ & & \\
Ethnicity & 19 & 11.2 \\
Black & 90 & 53.3 \\
Caucasian & 58 & 34.3 \\
Hispanic & 2 & 1.2 \\
Native American & & \\
$\quad$ & & \\
Gender & 8 & 4.7 \\
Female & 161 & 95.3 \\
Male & & \\
Study eye & & \\
Right & 86 & 50.9 \\
Left & 83 & 49.1 \\
\hline
\end{tabular}

Abbreviations: $\mathrm{OH}$, ocular hypertension; POAG, primary open-angle glaucoma.

${ }^{\text {a }}$ Percentage represents percentage of treated group using prostaglandin analog agent.

Table 2 Pearson's correlation $(r)$ between CH, CCT, DCT-IOP, and GAT-IOP

\begin{tabular}{lccccccc}
\hline & CCT & P-value & $\begin{array}{c}\text { DCT- } \\
\text { IOP }\end{array}$ & P-value & $\begin{array}{c}\text { GAT- } \\
\text { IOP }\end{array}$ & P-value \\
\hline $\begin{array}{l}\text { CH } \\
\quad \begin{array}{l}\text { Full sample } \\
(n=169)\end{array}\end{array}$ & 0.44 & $<0.001$ & -0.21 & 0.007 & -0.17 & 0.03 \\
$\begin{array}{l}\text { POAG cohort } \\
(n=81)\end{array}$ & 0.37 & $<0.001$ & -0.37 & $<0.001$ & -0.31 & 0.005 \\
$\begin{array}{l}\text { OH cohort } \\
(n=88)\end{array}$ & 0.35 & $<0.001$ & -0.36 & $<0.001$ & -0.35 & $<0.001$ \\
\hline
\end{tabular}

Abbreviations: $\mathrm{CCT}$, central corneal thickness; $\mathrm{CH}$, corneal hysteresis; DCT-IOP, intraocular pressure as measured by Pascal Dynamic Contour Tonometer; GAT-IOP, intraocular pressure as measured by Goldmann applanation tonometer; $\mathrm{OH}$, ocular hypertension; POAG, primary openangle glaucoma.

those parameters with significant $P$-values are listed in Table 4 . In univariate regression analyses, glaucoma diagnosis was associated with higher age and lower CCT, $\mathrm{CH}, \mathrm{CRF}$, IOP, and A1C. In multivariate analysis, higher age along with lower IOP and $\mathrm{CH}$ were the only variables independently associated with glaucoma diagnosis.

After dividing the sample into thin, intermediate, and thick CCT subgroups, mean $\mathrm{CH}$ was the only predictor variable that significantly differed between POAG and $\mathrm{OH}$ in all three CCT subgroups (Table 3). Moreover,

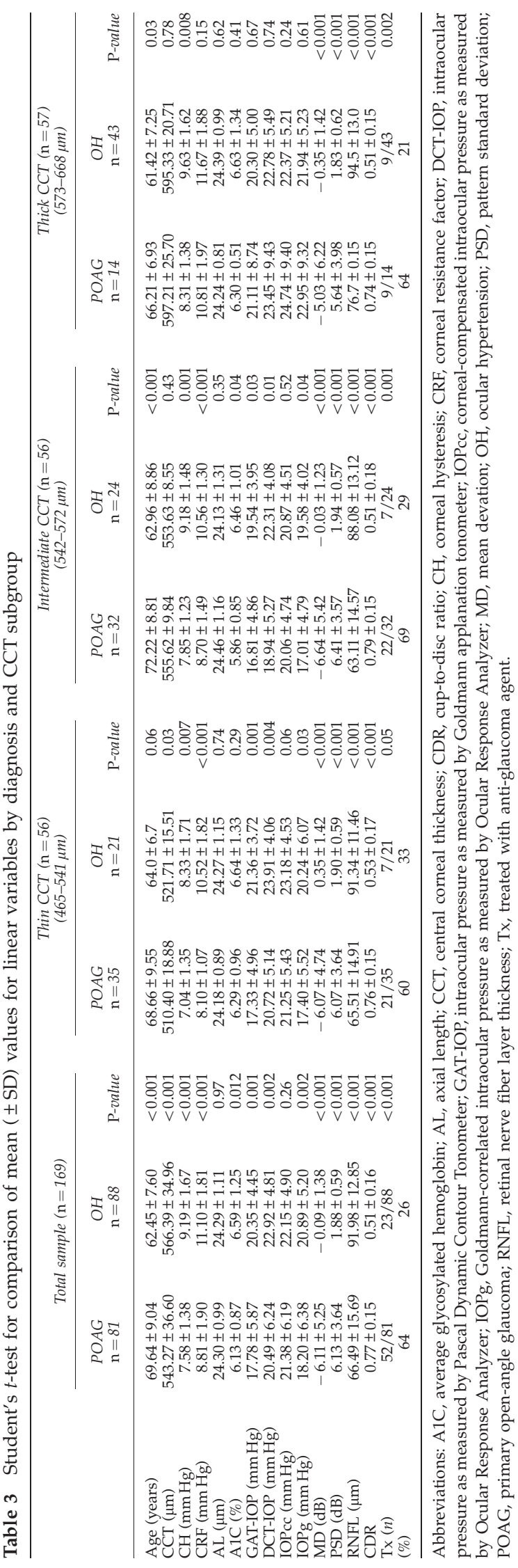


although mean $\mathrm{CH}$ increased with increasing CCT, mean $\mathrm{CH}$ remained significantly lower in POAG eyes compared with $\mathrm{OH}$ eyes within all CCT subgroups (Figure 1). In logistic regression analyses within the CCT groups, excluding variables related to glaucoma severity (MD, PSD, RNFL, CDR) and treatment, only four parameters were significantly associated with glaucoma diagnosis. Of these, $\mathrm{CH}$ was the only variable that was independently related to glaucoma diagnosis in all three CCT subgroups (Table 4 ). In addition, the $\beta$-coefficients for $\mathrm{CH}$ were higher in the CCT subgroups compared with the full sample.

When IOP-adjusted $\mathrm{CH}$ values were used in place of unadjusted $\mathrm{CH}$ values in regression analyses, $\beta$-coefficients for GAT-adjusted $\mathrm{CH}$, DCT-adjusted $\mathrm{CH}$, and, for comparison, unadjusted $\mathrm{CH}$ were: -0.57 , -0.53 , and -0.70 in the thin CCT subgroup; -0.53 , -0.59 , and -0.73 in the intermediate CCT group; and $-0.65,-0.62$, and -0.63 in the thick CCT subgroup. Thus, adjusting $\mathrm{CH}$ based on GAT-IOP and DCT-IOP did not provide additional diagnostic benefit compared with unadjusted $\mathrm{CH}$ values in this study.

\section{Discussion}

Although mean $\mathrm{CH}$ in the POAG eyes was significantly lower than that in the $\mathrm{OH}$ eyes, the relationship between
$\mathrm{CH}$ and diagnosis was compromised by considerable overlap in $\mathrm{CH}$ values between the diagnostic groups. These findings are consistent with other reports and underline the diagnostic promise as well as the shortcomings associated with $\mathrm{CH} .1,12,16$ After the study sample was divided into CCT tertiles, however, $\mathrm{CH}$ differences between POAG and $\mathrm{OH}$ were better delineated as shown in Figure 1. Specifically, while some overlap remained, the $\mathrm{CH}$ ranges within the subgroups appeared smaller for both POAG and $\mathrm{OH}$. Moreover, the $\beta$-coefficients for $\mathrm{CH}$ were higher in the CCT subgroups compared with the full sample, suggesting a more robust relationship between $\mathrm{CH}$ and diagnosis when $\mathrm{CCT}^{\prime} \mathrm{s}$ effect on $\mathrm{CH}$ was taken into account. Taken together, these findings indicate that combining CCT and $\mathrm{CH}$ enhances diagnostic precision compared with using either factor in isolation.

Additionally and in agreement with many other studies, ${ }^{1,2,4-8,12,14}$ we found that as CCT increases so does $\mathrm{CH}$, suggesting that corneal viscoelastic behavior is influenced by its thickness. Accordingly, in the thinner cornea eyes $(<542 \mu \mathrm{m})$, mean $\mathrm{CH}$ values were 7.0 for POAG and 8.3 for $\mathrm{OH}$, while the mean $\mathrm{CH}$ values in the intermediate CCT group $(542-572 \mu \mathrm{m})$ increased to 7.9 for POAG and 9.2 for OH. For eyes with thicker corneas $(>572 \mu \mathrm{m})$, the mean $\mathrm{CH}$ values rose to 8.3 and 9.6 for POAG and $\mathrm{OH}$, respectively. These findings

Table 4 Univariate and multivariate logistic regression analysis of factors associated with POAG

\begin{tabular}{|c|c|c|c|c|c|c|c|}
\hline & \multirow[t]{2}{*}{ Factor } & \multicolumn{3}{|c|}{ Univariate analysis } & \multicolumn{3}{|c|}{ Multivariate analysis } \\
\hline & & Estimate $\pm S E$ & P-value & $\beta$-coefficient & Estimate $\pm S E$ & P-value & $\beta$-coefficient \\
\hline \multicolumn{8}{|l|}{ All eyes } \\
\hline & Age & $0.02 \pm 0.004$ & $<0.001$ & 0.40 & $0.01 \pm 0.004$ & 0.005 & 0.26 \\
\hline & $\mathrm{A} 1 \mathrm{C}$ & $-0.09 \pm 0.037$ & 0.01 & -0.21 & & & \\
\hline & DCT-IOP & $-0.02 \pm 0.006$ & 0.002 & -0.24 & $-0.03 \pm 0.006$ & 0.001 & -0.31 \\
\hline & CRF & $-0.12 \pm 0.015$ & $<0.001$ & -0.53 & & & \\
\hline & ССТ & $-0.004 \pm 0.001$ & $<0.001$ & -0.32 & & & \\
\hline & $\mathrm{CH}$ & $-0.13 \pm 0.014$ & $<0.001$ & -0.45 & $-0.12 \pm 0.020$ & $<0.001$ & -0.41 \\
\hline \multicolumn{8}{|l|}{ Thin CCT group } \\
\hline & DCT-IOP & $-0.16 \pm 0.061$ & 0.01 & -0.50 & $-0.24 \pm 0.081$ & 0.002 & -0.75 \\
\hline & CRF & $-0.81 \pm 0.222$ & $<0.001$ & -0.92 & & & \\
\hline & $\mathrm{CH}$ & $-0.48 \pm 0.204$ & 0.02 & -0.43 & $-0.78 \pm 0.273$ & 0.004 & -0.70 \\
\hline \multicolumn{8}{|l|}{ Intermediate CCT group } \\
\hline & Age & $0.12 \pm 0.038$ & 0.002 & 0.64 & & & \\
\hline & DCT-IOP & $-0.15 \pm 0.068$ & 0.02 & -0.42 & $-0.21 \pm 0.084$ & 0.01 & -0.59 \\
\hline & CRF & $-0.91 \pm 0.261$ & $<0.001$ & -0.84 & & & \\
\hline & $\mathrm{CH}$ & $-0.76 \pm 0.275$ & 0.005 & -0.63 & $-0.87 \pm 0.335$ & 0.01 & -0.73 \\
\hline \multicolumn{8}{|l|}{ Thick CCT group } \\
\hline & Age & $0.09 \pm 0.046$ & 0.04 & 0.39 & & & \\
\hline & $\mathrm{CH}$ & $-0.76 \pm 0.301$ & 0.01 & -0.67 & $-0.72 \pm 0.307$ & 0.02 & -0.63 \\
\hline
\end{tabular}

Abbreviations: A1C, average glycosylated hemoglobin; CCT, central corneal thickness; $\mathrm{CH}$, corneal hysteresis; CRF, corneal resistance factor; DCT-IOP, intraocular pressure as measured by Pascal Dynamic Contour Tonometer; POAG, primary open-angle glaucoma.

Models based on probability of POAG diagnosis (eg, decreasing $\mathrm{CH}$ is associated with greater likelihood of POAG). 


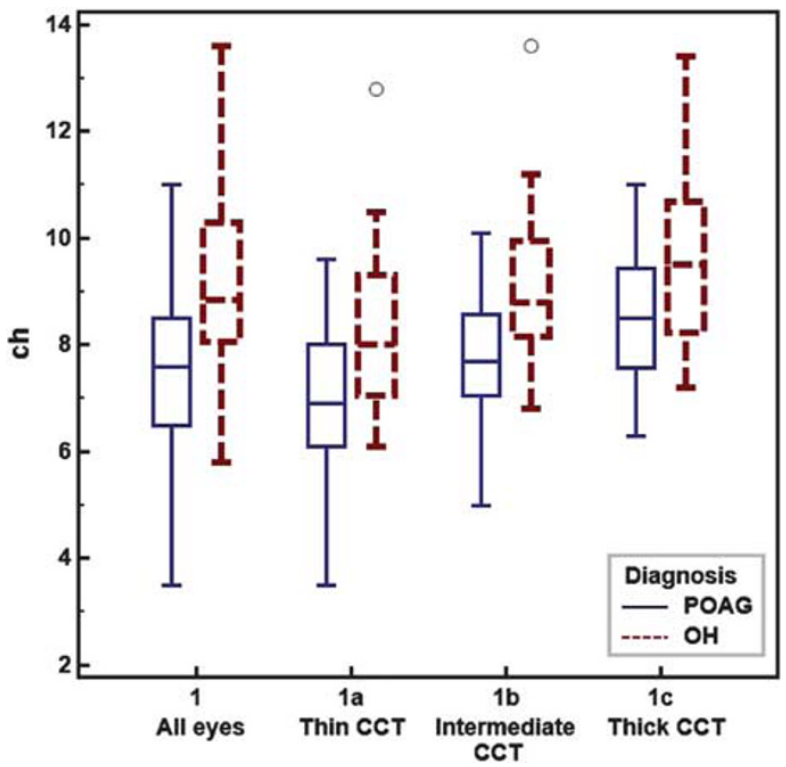

Figure 1 POAG vs $\mathrm{OH}: \mathrm{CH}$ differences in all eyes vs CCT subgroups.

suggest that the $\mathrm{CH}$ value alone, without consideration of the corneal thickness, could erroneously suggest or disguise the risk of glaucoma. For example, a $\mathrm{CH}$ value of 8.0 would suggest POAG in an eye with thicker CCT, but be more consistent with $\mathrm{OH}$ in an eye with thinner CCT. In addition, as seen in Figure 1, $\mathrm{OH}$ was rare when $\mathrm{CH}$ was below 7.0 but, when present, it was associated with thinner CCT. Conversely, POAG was rare when $\mathrm{CH}$ exceeded 10.0 but, when present, it was associated with thicker CCT.

Although it remains unclear whether lower mean $\mathrm{CH}$ in POAG eyes represents glaucoma-induced tissue remodeling, inherent risk for glaucoma, or both, findings from this study nonetheless support using $\mathrm{CH}$ and CCT together for glaucoma risk assessment. For instance, $\mathrm{OH}$ eyes that are initially characterized as higher risk, due to thinner CCT, may have their risk amplified further if their $\mathrm{CH}$ value is also low (as determined by CCT subgroup guidelines). In this case, the lower $\mathrm{CH}$ value may reflect greater biomechanical susceptibility and/or active tissue remodeling related to early stages of glaucoma pathophysiology. Alternatively, if the $\mathrm{CH}$ value is high in an eye with thinner CCT, the level of concern may be lessened as that eye may be better able to manage IOP-related biomechanical challenge. Conversely, in eyes considered at lower risk because of thicker CCT, a low $\mathrm{CH}$ might reflect increased biomechanical susceptibility or evolving tissue remodeling in that eye. In either case, that eye may deserve extra clinical attention. In summary, these findings suggest that combining $\mathrm{CCT}$ and $\mathrm{CH}$ for glaucoma risk assessment may be clinically useful. However, these preliminary findings do require further validation within longitudinal studies.

IOP is another factor known to influence $\mathrm{CH}$, with most studies reporting a significant inverse relationship between $\mathrm{CH}$ and IOP. ${ }^{1-11,27}$ Thus, as IOP increases, $\mathrm{CH}$ values systematically decrease, particularly when IOP values exceed the statistically normal range. ${ }^{27}$ Whether this phenomenon is physiological, an artifact of testing, or a combination of the two is debated, but a physiological relationship between $\mathrm{CH}$ and IOP appears reasonable if one considers that increasing IOP is associated with stiffening of the ocular coats (sclera/ cornea) ${ }^{23,24}$ and stiffer tissues have reduced capability to dampen further increases of IOP-related stress. ${ }^{23,24}$ Moreover, reduction in the eye's dampening capacity as IOP increases may play a role in explaining how IOP is related to the glaucomatous disease process considering that IOP-related load that is not absorbed by the ocular coats is likely transmitted to the weakest portion of the eye, the tissues within and around the optic nerve head. ${ }^{25}$ Alternatively, the $\mathrm{CH} / \mathrm{IOP}$ relationship may be related to the ORA's measurement technique. Because the ORA's escalation of air-jet pressure is more rapid in eyes with greater resistance (due to higher IOP and/or greater corneal rigidity), and because $\mathrm{CH}$ is a rate-dependent property, $\mathrm{CH}$ measurement may vary because of different rates of air-pressure challenge in eyes with different levels of IOP. However, given that some studies report that $\mathrm{CH}$ is lower in POAG vs normal eyes even when the IOP level is similar between the eyes, ${ }^{1,14}$ artifact from the ORA measurement process cannot fully explain lower $\mathrm{CH}$ in glaucomatous eyes.

We did not find that IOP-adjusted $\mathrm{CH}$ values provided any benefit compared with unadjusted $\mathrm{CH}$ values in this study. The reason for this may be related to the weak correlation between $\mathrm{CH}$ and IOP and/or the relatively small dynamic range of IOP in our data. Specifically, the 95\% confidence interval range for DCT-IOP (12.3$37.0 \mathrm{~mm} \mathrm{Hg}$ ) and GAT-IOP (9.9-31.6 mm Hg) was associated with a $\mathrm{CH}$ change of only 1.5 and $1.2 \mathrm{~mm} \mathrm{Hg}$, respectively, in this study. As a comparison, the $95 \%$ confidence interval range for CCT $(486-646 \mu \mathrm{m})$ was associated with a $\mathrm{CH}$ change of $3.2 \mathrm{~mm} \mathrm{Hg}$. Thus, in this study, adjusting for CCT was more important than adjusting for IOP. However, because $\mathrm{CH}$ values appear to be considerably influenced by very high levels of IOP, this finding may not apply in eyes with IOP $>32 \mathrm{~mm} \mathrm{Hg}$. In addition, it is likely that $\mathrm{CH}$ response to IOP is quite variable in individual patients, ${ }^{31}$ and thus IOP may have more substantial influence on $\mathrm{CH}$ in some patients compared with others. Further investigation is required to better understand the complex relationship between $\mathrm{CH}$ and IOP. 
In this study, the $\mathrm{OH}$ eyes exhibited higher mean IOP compared with the POAG eyes in the thin and intermediate CCT subgroups. The higher IOP in these eyes is likely due to differential treatment effect as more POAG eyes (64\%) than OH eyes (26\%) were being treated with topical glaucoma medications. To address these inter-group IOP differences, we adjusted for IOP within our multivariate regression models and found that $\mathrm{CH}$ remained significantly lower in POAG compared with $\mathrm{OH}$ even after adjusting for IOP. Moreover, had mean IOP been similar between the POAG and $\mathrm{OH}$ groups rather than being higher in our $\mathrm{OH}$ group, the $\mathrm{CH}$ differences between POAG and $\mathrm{OH}$ would be expected to be somewhat larger than what we found. Specifically, $\mathrm{CH}$ would be expected to increase if mean IOP in $\mathrm{OH}$ eyes was lower or, alternatively, $\mathrm{CH}$ would likely decrease with higher IOP in the POAG group. In either case, the difference in $\mathrm{CH}$ values between the groups would be expected to increase because of the underlying inverse relationship between IOP and $\mathrm{CH}$. Given the limited effect of IOP on $\mathrm{CH}$ in this study, however, the difference would likely be small.

Prior reports suggest that chronic use of prostaglandin analog (PA) agents may lead to thinner CCT, 32,33 and it is possible that these agents could also affect $\mathrm{CH}$ given their capacity to remodel extracellular matrix. ${ }^{34}$ Because more POAG than $\mathrm{OH}$ eyes were treated with PA agents in this study, it is possible that these agents may have influenced the results. In the extreme case, the $\mathrm{CH}$ differences between diagnostic groups in this study might be explained by PA-induced corneal structural alterations. Although this study cannot specifically address this possibility, it is clear that the IOPindependent effects of topical anti-glaucoma agents on corneal structural properties need further investigation as these relationships may have very important clinical implications.

Strengths of this study include its prospective protocol and the utilization of methods to maximize data accuracy. We also employed a simple and easily implemented strategy for combining $\mathrm{CH}$ and $\mathrm{CCT}$ to differentiate POAG from $\mathrm{OH}$. The primary limitations of this study are its cross-sectional design, its limited sample size, and the use of only two factors (CCT and IOP) to adjust $\mathrm{CH}$ values. In addition, we could not account for IOP fluctuation, glaucoma severity, or topical IOP-lowering treatment influences on corneal structure. Further, the study's generalizability is limited by our clinic-based sampling method, the primarily male sample, and the incomplete ethnicity and refractive error ranges. Additional studies are needed to address these factors.

In summary, our results show that mean $\mathrm{CH}$ increases with increasing CCT in both POAG and $\mathrm{OH}$ eyes, but $\mathrm{CH}$ remains lower in POAG vs $\mathrm{OH}$ eyes across the range of CCT. Furthermore, the association between $\mathrm{CH}$ and diagnosis is more robust within the CCT subgroups compared with the full sample, suggesting that combining $\mathrm{CH}$ and $\mathrm{CCT}$ may result in more precise risk assessment compared with using either factor in isolation. Although these findings are promising, additional study is needed to further validate and optimize $\mathrm{CH}$ utility in glaucoma risk assessment.

\section{Summary}

\section{What was known before}

- The diagnostic capability of $\mathrm{CH}$ is hampered by considerable overlap in values between glaucoma and non-glaucoma patients. $\mathrm{CH}$ is affected by a number of ocular and systemic factors, including CCT and IOP.

What this study adds

- Combining CCT with $\mathrm{CH}$ improves the diagnostic capability of both factors. Adjusting $\mathrm{CH}$ for IOP did not improve its diagnostic capability in this study.

\section{Conflict of interest}

The authors declare no conflict of interest.

\section{References}

1 Sullivan-Mee M, Billingsley SC, Patel AD, Halverson KD, Alldredge BR, Qualls C. Ocular Response Analyzer in subjects with and without glaucoma. Optom Vis Sci 2008; 85(6): 463-470.

2 Narayanaswarmy A, Su DH, Baskaran M, Tan AC, Nongpiur ME, Htoon HM et al. Comparison of Ocular Response Analyzer parameters in Chinese subjects with primary angle-closure and primay open-angle glaucoma. Arch Ophthalmol 2011; 129(4): 429-434.

3 Iordanidou V, Hamard P, Gendron G, Labbe A, Raphael M, Baudouin C. Modifications in corneal biomechanics and intraocular pressure after deep sclerectomy. J Glaucoma 2010; 19(4): 252-256.

4 Narayanaswarmy A, Chung RS, Wu R, Park J, Wong W, Saw $\mathrm{S}$ et al. Determinants of corneal biomechanical properties in an adult Chinese population. Ophthalmology 2011; 118: 1253-1259.

5 Kamiya K, Hagishima M, Fujimura F, Shimizu K. Factors affecting corneal hysteresis in normal eyes. Graefes Arch Clin Exp Ophthalmol 2008; 246: 1491-1494.

6 Touboul D, Roberts C, Kerautret J, Garra C, Maurice-Tison $\mathrm{S}$, Saubusse E et al. Correlations between corneal hysteresis, intraocular pressure, and corneal central pachymetry. J Cataract Refract Surg 2008; 34(4): 616-622.

7 Broman AT, Congdon NG, Bandeen-Roche K, Quigley HA. Influence of corneal structure, corneal responsiveness, and other ocular parameters on tonometric measurement of intraocular pressure. J Glaucoma 2007; 16(7): 581-588.

8 Kotecha A, Elsheikh A, Roberts CR, Zhu H, Garway-Heath DF. Corneal thickness- and age-related biomechanical 
properties of the cornea measured with the ocular response analyzer. Invest Ophthalmol Vis Sci 2006; 47(12): 5337-5347.

9 Kotecha A, Oddone F, Sinapis C, Elsheikh A, Sinapis D, Sinapis A et al. Corneal biomechanical characteristics in patients with diabetes mellitus. J Cataract Refract Surg 2010; 36(11): 1822-1828.

10 Lim L, Gazzard G, Chan YH, Fong A, Kotecha A, Sim EL et al. Cornea biomechanical characteristics and their correlates with refractive error in Singaporean children. Invest Ophthalmol Vis Sci 2008; 49(9): 3852-3857.

11 Ang GS, Bochmann F, Townend J, Azuara-Blanco A. Corneal biomechanical properties in primary open angle glaucoma and normal tension glaucoma. J Glaucoma 2008; 17(4): 259-262.

12 Detry-Morel M, Jamart J, Pourjavan S. Evaluation of corneal biomechanical properties with the Reichert Ocular Response Analyzer. Eur J Ophthalmol 2011; 21(2): 138-148.

13 Abitbol O, Bouden J, Doan S, Hoang-Xuan T, Gatinel D. Corneal hysteresis measured with the Ocular Response Analyzer in normal and glaucomatous eyes. Acta Ophthalmol 2010; 88(1): 116-119.

14 Mangouritsas G, Morphis G, Mourtzoukos S, Feretis E. Association between corneal hysteresis and central corneal thickness in glaucomatous and non-glaucomatous eyes. Acta Ophthalmol 2009; 87(8): 901-905.

15 Wells AP, Garway-Heath DF, Poostchi A, Wong T, Chan KC, Sachdev N. Corneal hysteresis but not corneal thickness correlates with optic nerve surface compliance in glaucoma patients. Invest Ophthalmol Vis Sci 2008; 49(8): 3262-3268.

16 Shah S, Laiquzzaman M, Mantry S, Cunliffe I. Ocular Response Analyzer to assess hysteresis and corneal resistance factor in low tension, open angle glaucoma and ocular hypertension. Clin Exp Ophthalmol 2008; 36(6): 508-513.

17 Congdon NG, Broman AT, Bandeen-Roche K, Grover D, Quigley HA. Central corneal thickness and corneal hysteresis associated with glaucoma damage. Am J Ophthalmol 2006; 141(5): 868-875.

18 De Moraes CV, Hill V, Tello C, Liebmann JM, Ritch R. Lower corneal hysteresis is associated with more rapid glaucomatous visual field progression. J Glaucoma 2012; 21(4): 209-213.

19 Anand A, De Moraes CG, Teng CC, Tello C, Liebmann JM, Ritch R. Corneal hysteresis and visual field asymmetry in open angle glaucoma. Invest Ophthalmol Vis Sci 2010; 51(12): 6514-6518.

20 Grise-Dulac A, Saad A, Abitbol O, Febbraro JL, Azan E, Moulin-Tyrode C et al. Assessment of corneal biomechanical properties in normal tension glaucoma and comparison with open-angle glaucoma, ocular hypertension, and normal eyes. J Glaucoma 2011; e-pub ahead of print 5 July 2011; doi:10.1097/IJG.0b013e318220daf0.
21 Ayala M. Corneal hysteresis in normal subjects and in patients with primary open-angle glaucoma and pseudoexfoliation glaucoma. Ophthalmic Res 2011; 46(4): 187-191.

22 Gordon MO, Beiser JA, Brandt JD, Heuer DK, Higginbotham EJ, Johnson CA et al. The Ocular Hypertension Treatment Study: baseline factors that predict the onset of primary open-angle glaucoma. Arch Ophthalmol 2002; 120(6): 714-720.

23 Johnson CS, Mian SI, Moroi S, Epstein D, Izatt J, Afshari NA. Role of corneal elasticity in damping of intraocular pressure. Invest Ophthalmol Vis Sci 2007; 48(6): 2540-2544.

24 Liu J, He X. Corneal stiffness affects IOP elevation during rapid volume change in the eye. Invest Ophthalmol Vis Sci 2009; 50(5): 2224-2229.

25 Burgoyne CF, Downs JC, Bellezza AJ, Suh JK, Hart RT. The optic nerve head as a biomechanical structure: a new paradigm for understanding the role of IOP-related stress and strain in the pathophysiology of glaucomatous optic nerve head damage. Prog Retin Eye Res 2005; 24(1): 39-73.

26 Sigal IA, Flanagan JG, Ethier CR. Factors influencing optic nerve head biomechanics. Invest Ophthalmol Vis Sci 2005; 46(11): 4189-4199.

27 Sullivan-Mee M, Katiyar S, Pensyl D, Halverson KD, Qualls C. Relative importance of factors affecting corneal hysteresis measurement. Optom Vis Sci 2012; 89(5): E803-E811.

28 Brandt JD. Central corneal thickness, tonometry, and glaucoma risk-a guide for the perplexed. Can J Ophthalmol 2007; 42(4): 562-566.

29 Hager A, Loge K, Fullhas MO, Schroeder B, Grossherr M, Wiegand $\mathrm{W}$. Changes in corneal hysteresis after clear corneal cataract surgery. Am J Ophthalmol 2007; 144(3): 341-346.

30 Boehm AG, Weber A, Pillunat LE, Koch R, Spoerl E. Dynamic contour tonometry in comparison to intracameral IOP measurements. Invest Ophthalmol Vis Sci 2008; 49(6): 2472-2477.

31 Sun L, Shen M, Wang J, Fang Z, Xu A, Fang H et al. Recovery of corneal hysteresis after reduction of intraocular pressure in chronic primary angle-closure glaucoma. Am J Ophthalmol 2009; 147(6): 1061-1066.

32 Harasymowycz PJ, Papamatheakis DG, Ennis M, Brady M, Gordon KD. Travoprost Central Corneal Thickness Study Group. Relationship between travoprost and central corneal thickness in ocular hypertension and open-angle glaucoma. Cornea 2007; 26(1): 34-41.

33 Zhong Y, Shen X, Yu J, Tan H, Cheng Y. The comparison of the effects of latanoprost, travoprost, and bimatoprost on central corneal thickness. Cornea 2011; 30(8): 861-864.

34 Lindsey JD, Kashiwagi K, Kashiwagi F, Weinreb RN. Prostaglandins alter extracellular matrix adjacent to human ciliary muscle cells in vitro. Invest Ophthalmol Vis Sci 1997; 38(11): 2214-2223. 